\title{
Incremental Displacement Collocation Method for the Evaluation of Tension Softening Curve of Mortar
}

\author{
R.K.L. Su ${ }^{*}$, H.H.N. Chen, A.K.H. Kwan \\ Department of Civil Engineering, The University of Hong Kong, Pokfulam Road, \\ Hong Kong, PRC
}

\begin{abstract}
The tension softening curve (TSC), showing the relationship between the cohesive tensile stress and crack opening displacement, is the constitutive law of the cohesive crack model. Due to the difficulties in measuring local deformations around the crack tip, the TSC is usually determined inversely from the global responses such as loaddeflection curve or load-crack mouth opening displacement curve of pre-notched specimens. However, the use of global responses alone in the inverse analysis usually causes problems that may affect the reliability and accuracy of the TSC which is basically a local material property. To overcome these limitations, an incremental displacement collocation method (IDCM) that is able to evaluate the TSC in a stepby-step manner is proposed in this paper. Both global and local responses of a prenotched mortar beam, which are measured using an electronic speckle pattern interferometry technique, are used in the displacement collocation process. Furthermore, the finite element model (FEM) is utilized to simulate the response of the beam. The TSCs evaluated in this study are verified through the comparisons of the global and local displacements as well as the fracture energy. A tri-linear curve was found to be the best approximation of the TSC of mortar.

Keywords: cohesive crack model; tension softening curve; displacement collocation; local response; electronic speckle pattern interferometry; finite element model; mortar
\end{abstract}




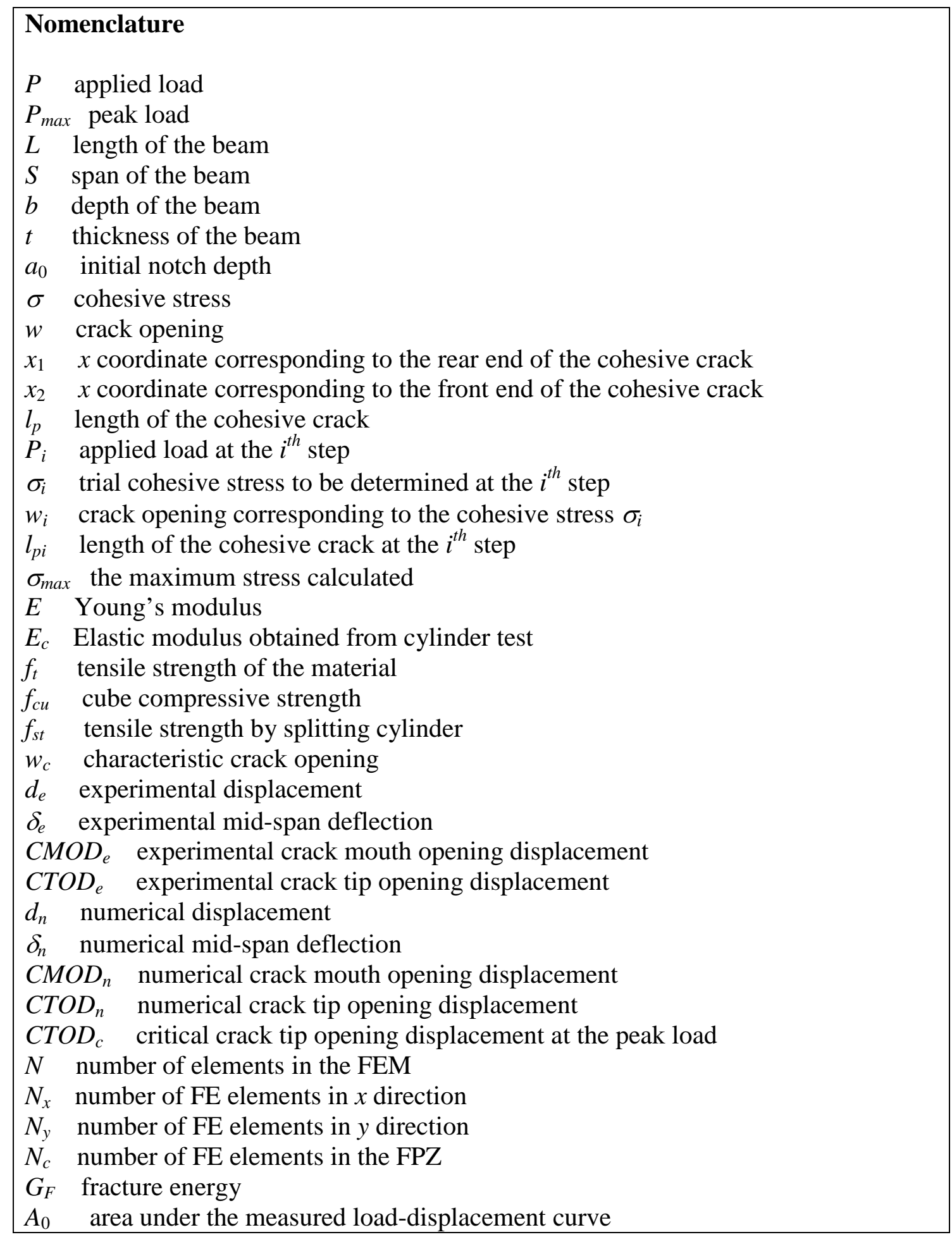




\section{Introduction}

Nonlinear fracture mechanics models have been proposed to explain the nonlinear fracture behavior of quasi-brittle materials, such as the cohesive crack model (CCM) or the fictitious crack model proposed by Hillerborg et al. [1]. According to the CCM, all the nonlinear behaviors in the fracture process zone (FPZ) are represented by a cohesive crack, and the crack propagation is dominated by the relationship of the cohesive stress versus crack opening, namely, the tension softening curve (TSC). The shape of the TSC has a significant influence on the computed results of a cracked beam in a finite element (FE) analysis; thus, a reliable estimation of the TSC is necessary.

Many efforts have been made to obtain the TSC. Ideally, the TSC can be obtained from uniaxial tension tests of the specimen, and some researchers [2] have produced uniaxial tension test configurations to analyze the tension-softening principles of concrete. However, the crack path may not be known a priori or the crack propagation may not be stable and symmetrical. Therefore, only the average value of the stress and crack opening can be obtained. It is difficult to make an accurate estimation of the TSC from a uniaxial tension test.

An alternative approach to evaluate the TSC is the inverse analysis based on the parametric fitting, which makes the numerical responses of a specimen agree with the experimental responses. Usually, notched beams or compact specimens are tested, and the experimental responses, such as load-deflection curve or load-crack mouth opening displacement (CMOD) curve, are used in the inverse analysis. The numerical responses of the specimen are obtained from a numerical model. As an input of the numerical model, the TSC is prescribed and obtained from an optimization procedure. Different assumptions on the shape of the TSC, such as linear [1], bilinear, tri-linear 
[3] and exponential [4-6] shapes, have been proposed. The bilinear curve is believed to be a reasonable model and has been most widely used in practice. Another approach to determine the TSC using the inverse method without a prior assumption on the shape of the TSC is based on the poly-linear approximation introduced by Kitsutaka [7] and applied by Kurihara et al. [8]. The TSC is determined from a series of loading states by correlating the numerical and experimental responses. For both aforementioned approaches, only the global responses of the specimen, such as loaddeflection or load-CMOD curve, are considered in the inverse analysis, taking no account of local responses.

The local responses of the beam were used in a hybrid inverse technique proposed by Shen and Paulino [9]. However, only the local response at one loading state in the post-peak stage was considered. The extracted TSC was only verified through the comparisons of the global response (load-CMOD curve) without checking the local responses at other loading states. Skoček and Stang [10] performed wedge splitting tests and inversely estimated the fracture parameters using the optically measured displacements. The softening curve is assumed to be piecewise and linear. The number of line segments in the softening curve has to be given a priori and the unknown parameters increase with the number of segments used.

An incremental displacement collocation method (IDCM) allowing an estimation of the TSC in a step-by-step manner is introduced in this study. At each numerical step, a trial cohesive stress forming the TSC is presumed and used along with the experimental crack opening to predict the cohesive stress in the FPZ. The trial cohesive stress is determined through the displacement collocation of the experimental displacements measured using the electronic speckle pattern interferometry (ESPI) technique and the numerical displacements from FE analysis. 
Compared with other available methods, the IDCM provides several advantages. First, the TSC is determined based on a piecewise-linear approximation without a prior assumption on the TSC shape. Thus, the most realistic TSC shape can be obtained. Second, the conventional elastic finite element program, which is commonly available, is capable of conducting the displacement collocation process. Third, both global and local responses of the specimen are considered in the displacement collocation, thereby reducing the extent of ill-posed conditions. Fourth, the estimated TSC satisfies the displacement requirements at all loading states. It is worth to note that for one single loading state, it is not difficult to find a TSC to satisfy the displacement requirements; however, the real TSC reflects the evolution of the crack and should satisfy the displacement requirements at all loading states. Finally, with only one unknown to be determined at each loading step, the IDCM offers a simple and stable estimation process of the TSC.

\section{Theoretical background of the IDCM}

\subsection{Theoretical background}

The determination of the TSC using the IDCM is facilitated through the integration of the CCM into the FEM. The basic assumptions adopted in the IDCM include

(1) The specimen is assumed to be isotropic and to behave in a linear elastic manner. All the nonlinear behavior is represented by a cohesive crack ahead of the notch tip.

(2) The cohesive crack begins to develop when the maximum tensile stress becomes equal to the tensile strength.

(3) The cohesive stresses in the FPZ are dominated by the extended TSC and the actual crack opening measured experimentally. 
(4) The trial cohesive stress follows a decreasing trend as the crack opening increases.

(5) The free surface effects [11] of a through thickness crack have been ignored. The three-dimensional cracked beam is simplified to be a two-dimensional beam in the FEM.

Above all, the theoretical background of the IDCM includes two aspects: the CCM and the principle of the incremental displacement collocation.

\subsubsection{The CCM}

It has been widely accepted that the CCM can provide a consistent and reasonable interpretation of the fracture behavior of a Mode I crack in quasi-brittle materials. In this model, the nonlinear behavior of all the crack regions is represented by the relationship between the cohesive stress $\sigma$ and the crack opening $w$ in the FPZ.

Fig. 1a and b show a sketch of a fully developed cohesive crack. Fig. 1b shows that at the point where $w=w_{c}$, the cohesive stress is zero; at the point where $w=0$, the cohesive stress equals the tensile strength $f_{t}$. For $0<w<w_{c}$, the cohesive stress is related to $w$ according to the cohesive law, as shown in Fig. 1c. For a cohesive crack with its front and rear ends at the locations $x=x_{2}$ and $x=x_{1}$, respectively, the length of the cohesive crack is

$$
l_{p}=x_{2}-x_{1}
$$

At the early loading stages of a test, a cohesive crack may not be fully developed; hence, the cohesive crack rear end $\left(x=x_{1}\right)$ coincides with the initial notch tip while the crack front $\left(x=x_{2}\right)$ can be determined from the experimental COD profile. As the crack opening displacement at the crack front $\left(x=x_{2}\right)$ is close to zero, the 
corresponding cohesive stress equals $f_{t}$. The crack opening at $x=x_{1}$ is smaller than $w_{c}$ until the notch tip is fully open ( $w \geq w_{c}$ ). Because the crack opening $w$ at all locations can be obtained directly from the experimental COD profile, the cohesive stress distribution within the FPZ can be evaluated once the piecewise-linear relationship of the TSC has been defined.

\subsubsection{Principle of the IDCM}

The parameters to be determined from the proposed IDCM include the piecewiselinear relationship of the TSC and the Young's modulus $E$ of the material. Through the load test of a pre-notched specimen and employing the sophisticated ESPI measurement technique, the global responses, such as the load-deflection and loadCMOD curves, and the local responses, including the complete COD profile, the location of crack front $\left(x_{2}\right)$ and hence $l_{p}$ at each of the loading steps, can be precisely determined. In this study, both the global and local responses are considered in the proposed IDCM. By matching the computed and measured displacements at various collocation points, the TSC and the Young's modulus can be evaluated.

In the proposed method, the TSC is determined in a step-by-step manner as shown in Fig.2. In each loading step, only one portion (or line-segment) of the TSC is evaluated. Except the first few steps that require determining both the Young's modulus $E$ and the ultimate tensile stress $f_{t}$, the subsequent steps, for example, the $i^{\text {th }}$ step, only requires finding the cohesive stress $\sigma_{i}$ at $x=x_{1}$ (i.e., the initial notch tip) of which the corresponding crack opening displacement is $w_{i}$. After finding the coordinates $\left(w_{i}, \sigma_{i}\right)$

on the TSC in the $i^{\text {th }}$ step, we can proceed to the next loading step. Repeating the aforementioned process, the entire TSC can be constructed incrementally with the increase of the loading steps. 


\subsection{Description of the methodology}

A flow chart of computational procedure for the evaluation of the TSC using the IDCM is presented in Fig. 3, and the details are described herein.

Step 1: Extraction of the experimental displacements

Using the ESPI techniques, precise displacements, including the mid-span deflection $\delta$, CMOD, COD profile and the position of crack front $\left(x_{2}\right)$, are determined.

\section{Step 2: Estimation of $f_{t}$ and $E$}

It is not easy to accurately estimate $f_{t}$ and $E$ of quasi-brittle materials from laboratory tests. Usually, a splitting cylinder test is performed to estimate $f_{t}$; however, Ghaffar et al. [12] indicated that $f_{t}$ obtained by a splitting cylinder is higher than that obtained from a uniaxial tension test and lower than that obtained from a flexural test. In the present study, the ultimate tensile strength $f_{t}$ will be determined inversely at the early loading stages.

At the early loading stages where the applied load $P$ is less than one-third of the peak load $P_{\text {max }}$, the crack opening $w$ is small as denoted by the open circles in Fig. 2a and b, and the length of FPZ is short. The cohesive stress in the FPZ can be assumed to be uniformly distributed and equal to $f_{t}$, which has negligible influence on the response of the beam. Next, the Young's modulus $E$ can be determined by comparing the computed and measured displacements. If the computed displacements are higher than the measured ones, a higher $E$ value should be used; conversely, if the computed displacements are lower than the test ones, a lower $E$ value should be chosen. 
Once the $E$ value has been estimated, $f_{t}$ can be evaluated. In the FE analysis, the applied load $P$ attempts to open the crack, while the cohesive stress tends to close the crack. If $f_{t}$ is too high, it may cause negative COD in the FPZ, even though $l_{p}$ is notably short; on the contrary, if $f_{t}$ is too low, to place the specimen in equilibrium, the local stress near the crack tip might be much higher than the $f_{t}$ assumed. Thus, a reasonable prediction of $f_{t}$ should satisfy two requirements, that a) the maximum calculated stress $\sigma_{\max }$ in the problem domain should be less than or equal to the $f_{t}$ assumed and there should be no abrupt change (or jumps) in the stress profile, and that b) positive CODs along the FPZ should be ensured.

Step 3: Determination of the length of the FPZ

The COD profile can be obtained from the relative horizontal displacement of two adjacent cross sections on both sides of the crack. Compared with the crack opening in the FPZ and pre-notch zone, the elastic deformations in the linear elastic zone are nearly zero. From the COD profile, the crack front in the FPZ, i.e., $x=x_{2}$ can be recognized. In addition, the initial notch tip can be taken as the crack rear in the FPZ, i.e., $x=x_{1}$. The length of the FPZ, $l_{p}$, can then be determined by Eq. (1).

Step 4: Calculation of the cohesive stress $\sigma(x)$

From the COD profile, crack opening $w(x)$ of the crack face nodes in the FPZ can be expressed in form of a polynomial:

$$
w(x)=\sum_{m} C_{m} x^{m}
$$

where $C_{m}(m=0,1,2 \ldots)$ are the coefficients of the polynomial and $x$ is the nodal coordinates on the crack face along the $x$ direction. 
At the $i^{\text {th }}$ loading step, the TSC constructed consists of $i$ number of line segments separated by $i$ nodal points $\left(w_{j}, \sigma_{j}\right), j=1,2 \ldots i$. All of the nodal points have been defined in the previous $i-1$ loading steps except the last one $\left(w_{i}, \sigma_{i}\right)$. In fact, $\sigma_{i}$ is the stress to be determined using the IDCM at the $i^{\text {th }}$ step. As the TSC is a decreasing function, the unknown stress $\sigma_{i}$ should satisfy the following requirement:

$$
\sigma_{i} \leq \sigma_{i-1}
$$

where $\sigma_{i-1}$ is the cohesive stress, which has been determined at the $i-1^{\text {th }}$ loading step. For $i=1$, from the previous sections, the ultimate tensile stress has been determined, and $\sigma_{0}$ equals to $f_{t}$.

By the linear interpolation, the nodal cohesive stress $\sigma(x)$ at the $j^{\text {th }}$ segment of the TSC can be expressed in terms of the crack opening by

$$
\sigma(x)=\sigma_{j-1}+\frac{\sigma_{j}-\sigma_{j-1}}{w_{j}-w_{j-1}}\left(w(x)-w_{j-1}\right)
$$

where $w(x)$ is the crack opening of the node considered, and $\left(w_{j-1}, \sigma_{j-1}\right)$ and $\left(w_{j}, \sigma_{j}\right)$ are the end coordinates of the $j^{\text {th }}$ line segment. The cohesive stresses $\sigma_{j-1}$ and $\sigma_{j}$ have been evaluated in the $j-1^{\text {th }}$ and $j^{\text {th }}$ steps, respectively. By assigning certain trial value for $\sigma_{i}$, which satisfies Eq. (3), and using Eq. (4), all of the nodal stresses along the FPZ can be obtained.

Step 5: Input the nodal stress $\sigma(x)$ into the FEM, the displacements at the collocation points can be calculated. The nodal cohesive stress $\sigma_{i}$ is selected only when the following two additional requirements are satisfied:

\section{Displacement requirement}

$$
\left|d_{n}-d_{e}\right|<\text { Tolerance }
$$


where $d_{n}$ and $d_{e}$ represent the computed and measured displacements, respectively, in terms of $\delta$, CMOD and CTOD.

To satisfy the above requirements, the nodal stress $\sigma_{i}$ is adjusted accordingly, if $d_{n}>d_{e}$, higher $\sigma_{i}$ should be chosen; on the contrary, if $d_{n}<d_{e}$, lower $\sigma_{i}$ should be used.

\section{Stress requirements}

All of the calculated stresses in the problem domain should not be greater than $f_{t}$, and the stress profile should be a smooth curve without sudden jumps or abrupt changes.

$$
\sigma_{\max } \leq f_{t}
$$

Prior to the formation of a fully developed cohesive crack, the nodal cohesive stress should be higher than zero; specifically,

$$
\sigma_{i}>0
$$

Step 6: If all the aforementioned requirements (Eqs. (5) to (7)) are satisfied, the analysis can proceed to the next loading step and repeat Step 2 to Step 5 until the crack opening at the initial notch tip reaches $w_{c}$ and $\sigma_{i}=0$.

One may find that the computed displacements agree with the experimental results only when $\sigma_{i}<0$, which implies that the crack rear end has propagated from the initial pre-notched tip and $l_{p}$ should be shorten accordingly by adjusting the location $x_{1}$ corresponding to the zero cohesive stress. Once the location of $x_{1}$ is obtained, the crack opening at $x_{1}$ is the characteristic crack opening $w_{c}$, and the analysis is terminated. 


\section{Implementation of the IDCM}

\subsection{Experiments}

\subsubsection{Mix proportions and specimens}

Two central-notched mortar beams were cast. Portland Cement CEM I 52.5N (BS EN197-1:2000) was used. The water: cement: fine aggregate ratio was 0.716: 1: 5.5 . The maximum size of the aggregate was $5 \mathrm{~mm}$. The size and configuration of the specimens were designed according to the guidelines proposed by the RILEM Technical committees on Fracture Mechanics of concrete [13]. The initial notch depth was $30 \mathrm{~mm}$, and the width of the notch was $3 \mathrm{~mm}$. The specimen size was $500 \mathrm{~mm} \times$ $100 \mathrm{~mm} \times 40 \mathrm{~mm}$, and the span of the beam was $400 \mathrm{~mm}$. To obtain the material properties, three cubes and two cylinders were created. After being cast and cured, the specimens were placed in air (temperature: $20 \pm 2^{\circ} \mathrm{C}$; relative humidity: $75-85 \%$ ) until the date of testing. According to the Hong Kong construction standard, the cubes were tested to determine the cube compressive strength $f_{c u}$, and the cylinders were tested to obtain the static modulus of elasticity in compression $E_{c}$ and the splitting tensile strength $f_{s t}$. The material properties $f_{c u}, E_{c}$ and $f_{s t}$ were found to be $29.2 \mathrm{MPa}, 19.5 \mathrm{GPa}$ and 2.25 MPa, respectively.

\subsubsection{Experimental setup and test procedures}

Using an MTS bend fixture, three-point bend tests were carried out at 28 days after casting the specimens. The experimental setup is presented in Fig. 4a. In the test, displacement control with a loading rate of $0.01 \mathrm{~mm} / \mathrm{min}$ of the jack displacement was used, and the jack was moved upward to apply load on the beam. The crack growth was well controlled by a closed-loop servo hydraulic control system. As pre-notched beams were employed in the test, the main crack propagated along the notch direction. 
Approximately 8 to 11 min were required to reach the peak load. The mid-span deflection and CMOD were measured by LVDTs and a clip gauge, respectively. The complete load-deflection and load-CMOD curves were recorded by a data logger. To measure the mid-span deflection without support disturbances, an additional frame was located on the top of the beam to act as a reference datum of LVDTs (see Fig. 4b). A 3D ESPI system (Q300 produced by Dantec-Ettemeyer) was used to measure the surface deformation at the mid-span of the beams. The specifications of Q300 system are tabulated in Table 1 . In the present study, the measuring area is about $170(\mathrm{H}) \times$ $130(\mathrm{~V}) \mathrm{mm}^{2}$. The measurement sensitivity of the ESPI system depends on the length of illumination arms, the object distance and the laser wavelength. With longer illumination arms, shorter object distance and shorter laser wavelength, the measurement sensitivity will be enhanced. With the present test setup, it is already sufficient to achieve a displacement resolution of $0.2 \mu \mathrm{m}$.

At each loading state, speckle pattern on the measuring surface was captured by an ESPI sensor. The post-processing software ISTRA was used to convert ESPI raw data to in-plane displacement distributions.

To ensure the reliability of the experimental results and acquire sufficient data for further numerical analysis, the following testing procedures are recommended.

(1) To eliminate the influence of rigid-body movements of the specimen, gypsum packing at the loading point and supports is necessary.

(2) Because the gypsum packing may develop some friction between the specimen surface and the rollers, steel plates are placed between the gypsum packing and the roller supports to allow the rollers to roll freely as the specimen deforms. 
(3) Preload should be less than $1 / 5$ of the peak load to avoid damage to the specimen before the test.

(4) Fringe patterns on the measuring surface are recorded by the ESPI sensor in a step-by-step manner. When using short illumination arms and a camera position $=350 \mathrm{~mm}$, a record should be taken when 2-3 fringes appear on each side of the notch.

(5) The deformation information at the early loading stages can only be used to estimate $f_{t}$ and $E$ but not the variation of the TSC. At the early loading stages, e.g., when CTOD $<3 \mu \mathrm{m}$, the FPZ is short enough that the non-uniform distribution of cohesive stress has a little influence on the deformations at the collocation points and can be neglected.

(6) To obtain the complete set of deformation information of a fully developed cohesive crack for the subsequent analysis, the test can only be terminated when the post-peak load is lower than $1 / 3$ of the peak load.

\subsubsection{Experimental results}

\section{Load-deflection and load-CMOD curves}

The two beam specimens, namely, Units 1 and 2 were tested. From the data logger readings, the $P$ - $\delta$ and $P$-CMOD curves of the two specimens were obtained, as shown in Fig. 5 and tabulated in Tables 2 and 3. The displacement information was used in the subsequent numerical analysis for extracting the TSC.

COD profile 
Full-field surface deformations at the mid-span of the beam were obtained by the ESPI technique. From the displacement data, the COD profile along the crack path was extracted.

The measuring surface is shown in Fig. 6a. Three zones (the linear elastic zone, the FPZ and the pre-notch zone) are also presented in the figure. From the theoretical point of view, in the linear elastic zone, there is no crack; thus, the COD should be zero. In the FPZ, because the crack initiates and propagates, the COD is higher than zero (see Fig. 6b). The location from which the COD starts to increase can be regarded as the crack front of the cohesive crack. By assuming the rear end of the cohesive crack is at the initial notch tip, the length of the cohesive crack can be obtained. Furthermore, the crack opening $w$ in the FPZ can be expressed into a polynomial in terms of $x$ coordinates on the crack face (see Fig. 6b). For a small FPZ, the polynomial fitting can be quadratic; while for a large FPZ, a higher order polynomial should be used.

\subsection{FEM}

The three point bend test was simulated using an FEM program written in FORTRAN. According to the symmetry of the specimen, only one half of the beam was analyzed. The specimen configuration and FE meshes are illustrated in Fig. 7. A range of 225 to 900 9-node hybrid elements [14] were used in the analysis. Because a steel plate was provided between the roller and the specimen, the applied force was treated as a uniformly distributed load. The bulk material was considered to be linear elastic. The Young's modulus $E=25560 \mathrm{MPa}$ and $25532 \mathrm{MPa}$ for Units 1 and 2, respectively, and the tensile strength $f_{t}=3.3 \mathrm{MPa}$ were determined by the IDCM; the detailed discussions are presented in Section 3.3. The Poisson's ratio $v$ was assumed to be 0.2. 


\subsubsection{Effect of mesh density}

To study the effect of mesh density, FE models with different numbers of elements were built. Because the cohesive crack is along the notch line, only the mesh in the $x$ direction is refined. Setting the element number in the $y$ direction $N_{y}=25$, the element number in $x$ direction $N_{x}=9,18$, and 36, corresponding to total numbers of elements $N=225,450$ and 900, respectively. At the peak load of Unit 2, the 2D meshes of one half of the specimen with $N=225,450$ and 900 are depicted in Figs. 7a, 7b and 7c, respectively.

The computed COD and stress profiles with different mesh densities are shown in Figs. 8a and 8b. Different numbers of elements yield similar results, and thus all of the meshes used are considered to be sufficiently fine.

The medium dense FE mesh with total number of elements $N=450$ was chosen for the subsequent numerical analysis. The numbers of elements in the $x$ and $y$ directions were $N_{x}=18$ and $N_{y}=25$, respectively. As the increase of the loading and propagation of the crack, the mesh densities in the three zones were adjusted, while the total element number remained unchanged.

\subsubsection{Effect of the cohesive stress $\sigma_{i}$}

Taking Unit 2 as an example and considering the peak load stage (Step 5), the FE mesh of the specimen is shown in Fig. 7b. By assigning different values to the cohesive stress $\sigma_{5}$, different COD and stress profiles as shown in Figs. 9a and 9b, respectively, are obtained. The higher the value of $\sigma_{5}$ that was chosen, the lower the crack opening was. In addition, this cohesive stress value affects the shape of the stress profiles along the center of the specimen. An appropriate stress value $\left(\sigma_{5}=1.6\right.$ 
$\mathrm{MPa})$ produced a smooth curve, while a higher value $\left(\sigma_{5}=2.6 \mathrm{MPa}\right)$ or lower value $\left(\sigma_{5}=0.6 \mathrm{MPa}\right)$ created abrupt changes and sudden jumps in the stress profiles. Therefore, the numerical results are quite sensitive to the selected value of the cohesive stress $\sigma_{i}$. This additional stress requirement is useful to eliminate the inappropriately assumed cohesive stresses and is of benefit for improving the accuracy of the IDCM.

\subsection{Determination of the TSCs of the two specimens}

Using the IDCM, the ultimate tensile strength and Young's modulus of the two specimens were determined. The tensile strength $f_{t}=3.3 \mathrm{MPa}$ obtained from the IDCM is about one third higher than $f_{s t}=2.25 \mathrm{MPa}$ from splitting tensile tests. Meanwhile, the Young's modulus E (=25.7 GPa and 25.5 GPa for Units 1 and 2 respectively) obtained from the IDCM is about one fourth higher than the static modulus of elasticity $E_{c}$ (= $\left.19.5 \mathrm{GPa}\right)$ obtained from cylinder tests. This discrepancy may be due to different compact and curing conditions of the beams and cylinders as well as different geometries and boundary conditions.

Fig. 10 shows the estimated TSCs for both specimens. The characteristic crack openings $w_{c}$ for Units 1 and 2 are $72.9 \mu \mathrm{m}$ and $87.5 \mu \mathrm{m}$, respectively. The ratio of $w_{c} \cdot f_{t}$ to the fracture energy $G_{F}(81.78 \mathrm{~N} / \mathrm{m})$ is approximately 3.24 , which is comparable to 3.6 as suggested by Petersson [15].

It is found that, at the peak load, the cohesive stresses at the initial notch tip are about 1.65 MPa $\left(0.5 f_{t}\right)$ and the corresponding crack opening $w$ is $15 \mu \mathrm{m}$. When $w$ increases from zero to $25 \mu \mathrm{m}$, the cohesive stresses drop quickly from $f_{t}$ to $0.8 \mathrm{MPa}$ for Unit 1 and $1 \mathrm{MPa}$ for Unit 2, which correspond to $0.25 f_{t}$ and $0.33 f_{t}$ respectively. Such stress levels closely agree with the values of $1 / 4 f_{t}$ suggested by Wittmann et al. [16] and 
$1 / 3 f_{t}$ proposed by Pettersson [15] for the break-point of the bilinear curve. From that point, the stress decreases gradually until $w$ increases to $75 \mu \mathrm{m}$ for Unit 1 and $60 \mu \mathrm{m}$ for Unit 2; next, the stress decreases rapidly to zero. Thus, the tail parts of the TSCs revealed in the present study are not as long as those in the models proposed in previous reports $[16,17]$. It is noted that many studies [18-20] reveal that the tail part of the softening curve exhibits a boundary effect and the tail of the curve would become longer with the increase of the specimen size. However, the investigation of size and boundary effects is beyond the scope of the present study. The TSC determined herein is particularly applicable to the specimen size adopted in this study. Referring to the aforementioned assumptions on the shape of the TSC, such as linear, bi-linear, tri-linear and exponential curves, a tri-linear curve was found to be the best approximation of mortar according to the present results.

\subsection{Comparison and verifications of the results}

\subsubsection{Comparisons of $P-\delta, P-C M O D$ and $P-C T O D$ curves}

As described in Section 2.2, the TSC could be constructed incrementally using the IDCM. At each loading step, the computed displacements were matched with the experimental results to determine a point on the TSC. When the loading step reached Step 12 for Unit 1 (or Step 9 for Unit 2), a fully-developed TSC has been evaluated. In the subsequent loading steps, the cohesive stress distribution in the FPZ was estimated by putting the experimental COD into the calibrated TSC obtained from the previous loading steps. By integrating the estimated cohesive stress distribution into the FEM, the displacements in the subsequent loading steps could be computed.

Fig. 11 compares the computed $P-\delta, P$-CMOD and P-CTOD curves with the measured results. Two kinds of computed results are presented. The solid circles in 
the beginning part of the curves represent the calibrated displacements obtained from the IDCM; the hollow circles in the latter part of the curves represent the predicted displacements for validating the calibrated TSC. Good agreement can be observed in all the comparisons, demonstrating the effectiveness and accuracy of the proposed method. From the P-CTOD curves, it can be found that, at the peak load, the critical crack opening displacement $C T O D_{c}$ is approximately $15 \mu \mathrm{m}$.

\subsubsection{Comparisons of the facture energy $G_{F}$}

As confirmed by Karihaloo and his co-workers [21-23], the specific fracture energy of concrete can be used as a size-independent material property for a beam with a given beam span-to-depth ratio and notch-to-beam depth ratio. Therefore the TSCs are further verified through the comparisons of the fracture energy $G_{F}$. There are two ways to estimate the fracture energy $G_{F}$.

a) One method is from the entire $P$ - $\delta$ curve by

$$
G_{F}=\frac{A_{0}}{\left(b-a_{0}\right) t}
$$

where $A_{0}$ is the area under the measured load-displacement curve, $b$ and $t$ are the depth and thickness of the beam, respectively, and $a_{0}$ is the initial notch depth.

b) Another approach is from the area under the TSC by

$$
G_{F}=\int_{0}^{w_{c}} \sigma(w) d w
$$

where $w_{c}$ is the characteristic crack opening corresponding to zero cohesive stress.

The comparisons of the fracture energy obtained from the two methods are presented in Table 4. The values from the areas under the TSCs are different with those from $P$ $\delta$ curves by 1.35 and $0.04 \mathrm{~N} / \mathrm{m}$ for Units 1 and 2, respectively. Excellent agreement of 
the fracture energy verifies the accuracy of the TSCs evaluated and the efficiency of the IDCM.

\section{Conclusions}

IDCM, which combines FEM analysis with the actual deformation field measured using the ESPI technique, was introduced to determine the TSC of mortar. The IDCM was implemented through experiments on pre-notched mortar beams and the FEM simulation. Numerical analysis indicates that the FE meshes used in this study are sufficiently fine to obtain consistent results. The assumed trial cohesive stress at the pre-notched tip can significantly affect the numerical results. Thus, it is crucial to choose an appropriate stress value at each loading step. The tensile strength $f_{t}$ and Young's modulus $E$ of two specimens can be determined at the early loading stages. The estimated TSCs show reasonable shapes in comparison to the models proposed by previous researchers. The TSCs are verified through the comparisons of $P$ - $\delta$ curves, $P$-CMOD curves, $P$-CTOD curves in which the displacements predicted using a fullydeveloped TSC agree well with the experimental results. Furthermore, the fracture energy obtained from the TSCs and that from $P-\delta$ curves are compared and, again, excellent agreement is found. A tri-linear curve is found to be the best approximation of the TSC of mortar.

\section{Acknowledgments}

The research described here was supported by the Research Grants Council of the Hong Kong SAR (Project No. HKU7166/08E) and The University of Hong Kong through Small Project Funding 2009-2010. 


\section{References}

[1] Hillerborg A, Modéer M and Petersson PE. Analysis of crack formation and crack growth in concrete by means of fracture mechanics and finite elements. Cement and Concrete Research 1976; 6: 773-781.

[2] Cornelissen HAW, Hordijk DA and Reinhardt HW. Experimental determination of crack softening characteristics of normalweight and lightweight concrete. HERON 1986; 31: 45-56.

[3] Liaw BM, Jeang FL, Du JJ, Hawkins NM and Kobayashi AS. Improved nonlinear model for concrete fracture. Journal of Engineering Mechanics 1990; 116: 429-445.

[4] Foote RML, Mai YW and Cotterell B. Crack-growth resistance curves in strainsoftening materials. Journal of the Mechanics and Physics of Solids 1986;34: 593-607. [5] Reinhardt HW. Crack softening zone in plain concrete under static loading. Cement and Concrete Research 1985; 15: 42-52.

[6] Gopalaratnam VS and Shah SP. Softening response of plain concrete in direct tension. Journal of the American Concrete Institute 1985; 82: 310-323.

[7] Kitsutaka Y. Fracture parameters by polylinear tension-softening analysis. Journal of Engineering Mechanics 1997; 123: 444-450.

[8] Kurihara N, Kunieda M, Kamada T, Uchida Y and Rokugo K. Tension softening diagrams and evaluation of properties of steel fiber reinforced concrete. Engineering Fracture Mechanics 2000; 65: 235-245.

[9] Shen B and Paulino GH. Direct extraction of cohesive fracture properties from digital image correlation: a hybrid inverse technique. Experimental Mechanics 2011; 51: 143-163.

[10] Skocek J and Stang H. Application of optical deformation analysis system on wedge splitting test and its inverse analysis. Materials and Structures 2010; 43: 63-72. 
[11] Leung AYT and Su RKL. Order of the singular stress fields of through-thickness cracks. International Journal of Fracture 1996; 75: 85-93.

[12] Ghaffar A, Chaudhry MA and Kamran Ali M. A new approach for measurement of tensile strength of concrete. Journal of Research (Science) 2005; 16: 1-9.

[13] RILEM. TC50-FMC fracture mechanics of concrete, determination of the fracture energy of mortar and concrete by means of three-point bend tests on notched beams. Materials and Structures 1985; 18: 287-290.

[14] Sze KY, Fan H and Chow CL. Elimination of spurious pressure and kinematic modes in biquadratic nine-node plane element. International Journal for Numerical Methods in Engineering 1995; 38: 3911-3932.

[15] Petersson PE. Crack growth and development of fracture zones in plain concrete and similar materials. Report No. TVBM 1006. Division of Building Materials, LTH, Lund university 1981.

[16] Wittmann FH, Rokugo K, Brühwiler E, Mihashi H and Simonin P. Fracture energy and strain softening of concrete as determined by means of compact tension specimens. Materials and Structures 1988; 21: 21-32.

[17] Guinea GV, Planas J and Elices M. A general bilinear fit for the softening curve of concrete. Materials and Structures 1994; 27: 99-105.

[18] Hu X and Wittmann FH. Fracture energy and fracture process zone. Materials and Structures 1992; 25: 319-326.

[19] Kwon SH, Zhao Z, Shah SP. Effect of specimen size on fracture energy and softening curve of concrete: part II. Inverse analysis and softening curve. Cement and Concrete Research 2008; 38: 1061-1069.

[20] Bretschneider N, Slowik V, Villmann B and Mechtcherine V. Boundary effect on the softening curve of concrete. Engineering Fracture Mechanics 2011; 78: 2896-2906. 
[21] Karihaloo BL, Abdalla HM and Imjai T. A simple method for determining the true specific fracture energy of concrete. Magazine of Concrete Research 2003; 55: 471-481.

[22] Abdalla HM and Karihaloo BL. Determination of size-independent specific fracture energy of concrete from three-point bend and wedge splitting tests. Magazine of Concrete Research 2003; 55: 133-141.

[23] Abdalla HM and Karihaloo BL. A method for constructing the bilinear tension softening diagram of concrete corresponding to its true fracture energy. Magazine of Concrete Research 2004; 56: 597-604. 
Table 1 Technical specifications of Q300 system

\begin{tabular}{|l|l|}
\hline Displacement accuracy & $0.05-1 \mu \mathrm{m}$ adjustable \\
\hline CCD-resolution & $1392(\mathrm{H}) \times 1040(\mathrm{~V})$ pixels \\
\hline Measuring range & $\begin{array}{l}\text { Adjustable, } 10-100 \mu \mathrm{m} \text { per step depending on measuring } \\
\text { direction }\end{array}$ \\
\hline Measuring area & Up to $200 \times 300 \mathrm{~mm}^{2}$ with built in illumination \\
\hline Working distance & Variable, $0.2 \ldots 1.0 \mathrm{~m}$ \\
\hline Laser (built in) & Diode, $2 \times 75 \mathrm{~mW}, \lambda=785 \mathrm{~nm}$ \\
\hline
\end{tabular}

Table 2 Experimental results of Unit 1: $f_{t}=3.3 \mathrm{MPa}$, $E=25650 \mathrm{MPa}$ and $a_{0}=30 \mathrm{~mm}$

\begin{tabular}{cccccc}
\hline $\begin{array}{c}\text { Step } \\
i\end{array}$ & $\begin{array}{c}P \\
(\mathrm{kN})\end{array}$ & $\begin{array}{c}l_{p} \\
(\mathrm{~mm})\end{array}$ & $\begin{array}{c}\delta_{e} \\
(\mu \mathrm{m})\end{array}$ & $\begin{array}{c}C M O D_{e} \\
(\mu \mathrm{m})\end{array}$ & $\begin{array}{c}C T O D_{e} \\
(\mu \mathrm{m})\end{array}$ \\
\hline 1 & 0.360 & 0.5 & 6.6 & 3.2 & 0.7 \\
2 & 0.455 & 1.5 & 10.1 & 5.9 & 1.3 \\
3 & 0.779 & 6 & 16.8 & 10.4 & 2.5 \\
4 & 1.027 & 8 & 26.1 & 15.5 & 4.1 \\
5 & 1.220 & 14 & 33.0 & 21.0 & 6.0 \\
6 & 1.430 & 19 & 41.2 & 26.7 & 9.7 \\
7 & 1.470 & 29 & 49.3 & 35.2 & 15.7 \\
8 & 1.310 & 38 & 64.3 & 49.8 & 26.0 \\
9 & 1.110 & 46 & 83.6 & 68.2 & 39.5 \\
10 & 0.922 & 51 & 102.5 & 87.8 & 53.7 \\
11 & 0.741 & 52 & 133.6 & 117.8 & 75.9 \\
12 & 0.511 & 54 & 163.6 & 150.3 & 99.5 \\
\hline
\end{tabular}

Table 3 Experimental results of Unit 2: $f_{t}=3.3 \mathrm{MPa}$, $E=25532 \mathrm{MPa}$ and $a_{0}=30 \mathrm{~mm}$

\begin{tabular}{cccccc}
\hline $\begin{array}{c}\text { Step } \\
i\end{array}$ & $\begin{array}{c}P \\
(\mathrm{kN})\end{array}$ & $\begin{array}{c}l_{p} \\
(\mathrm{~mm})\end{array}$ & $\begin{array}{c}\delta_{e} \\
(\mu \mathrm{m})\end{array}$ & $\begin{array}{c}C M O D_{e} \\
(\mu \mathrm{m})\end{array}$ & $\begin{array}{c}C T O D_{e} \\
(\mu \mathrm{m})\end{array}$ \\
\hline 1 & 0.336 & 0.5 & 7.0 & 3.4 & 0.6 \\
2 & 0.660 & 4 & 17.0 & 8.8 & 1.9 \\
3 & 0.975 & 8 & 27.0 & 14.9 & 3.4 \\
4 & 1.267 & 15 & 38.0 & 22.0 & 7.3 \\
5 & 1.416 & 25 & 54.0 & 32.6 & 14.9 \\
6 & 1.340 & 32 & 68.0 & 44.8 & 23.8 \\
7 & 1.110 & 43 & 87.0 & 66.6 & 39.1 \\
8 & 0.957 & 48 & 102.0 & 84.1 & 52.4 \\
9 & 0.608 & 53.5 & 132.0 & 115.4 & 76.7 \\
\hline
\end{tabular}

Table 4 Comparisons of fracture energy $(\mathrm{N} / \mathrm{m})$ estimated using different methods

\begin{tabular}{ccc}
\hline & Unit 1 & Unit 2 \\
\hline$P$ - $\delta$ Curves & 85.34 & 76.91 \\
The TSCs & 86.69 & 76.87 \\
Discrepancy & 1.35 & 0.04 \\
\hline
\end{tabular}


a

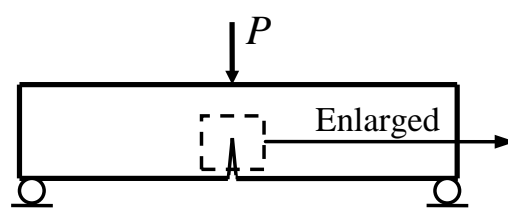

b

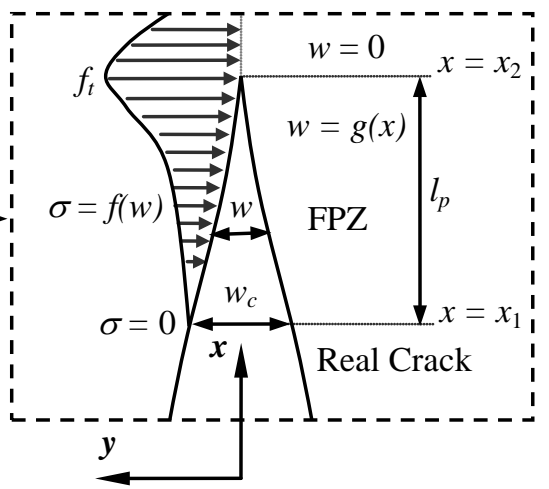

C

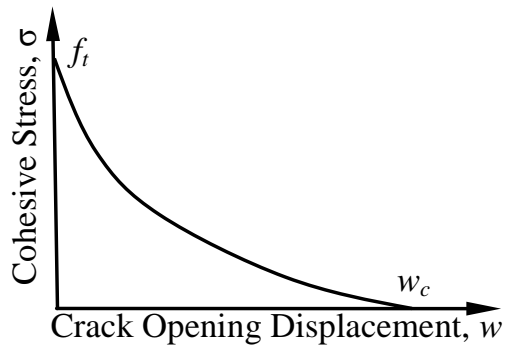

Fig. 1. (a) Sketch of a crack configuration. (b) Sketch of a cohesive crack. (c) Cohesive law.

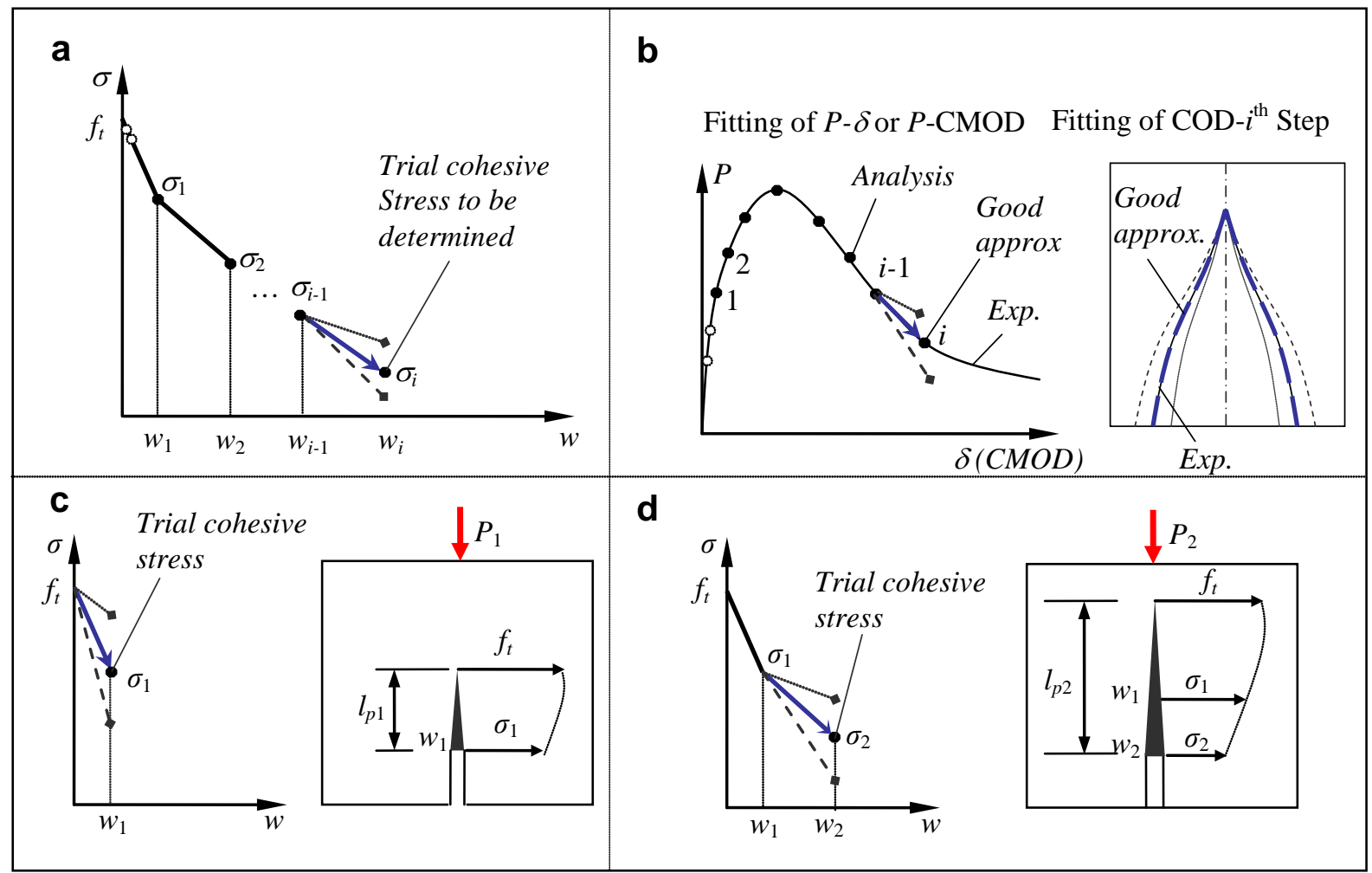

Fig. 2. The principle of the IDCM: (a) incremental construction of the TSC; (b) displacement collocation for the estimation of the trial stress; (c) estimation of the $1^{\text {st }}$ trial stress $\sigma_{1}$; (d) estimation of the $2^{\text {nd }}$ trial stress $\sigma_{2}$. 


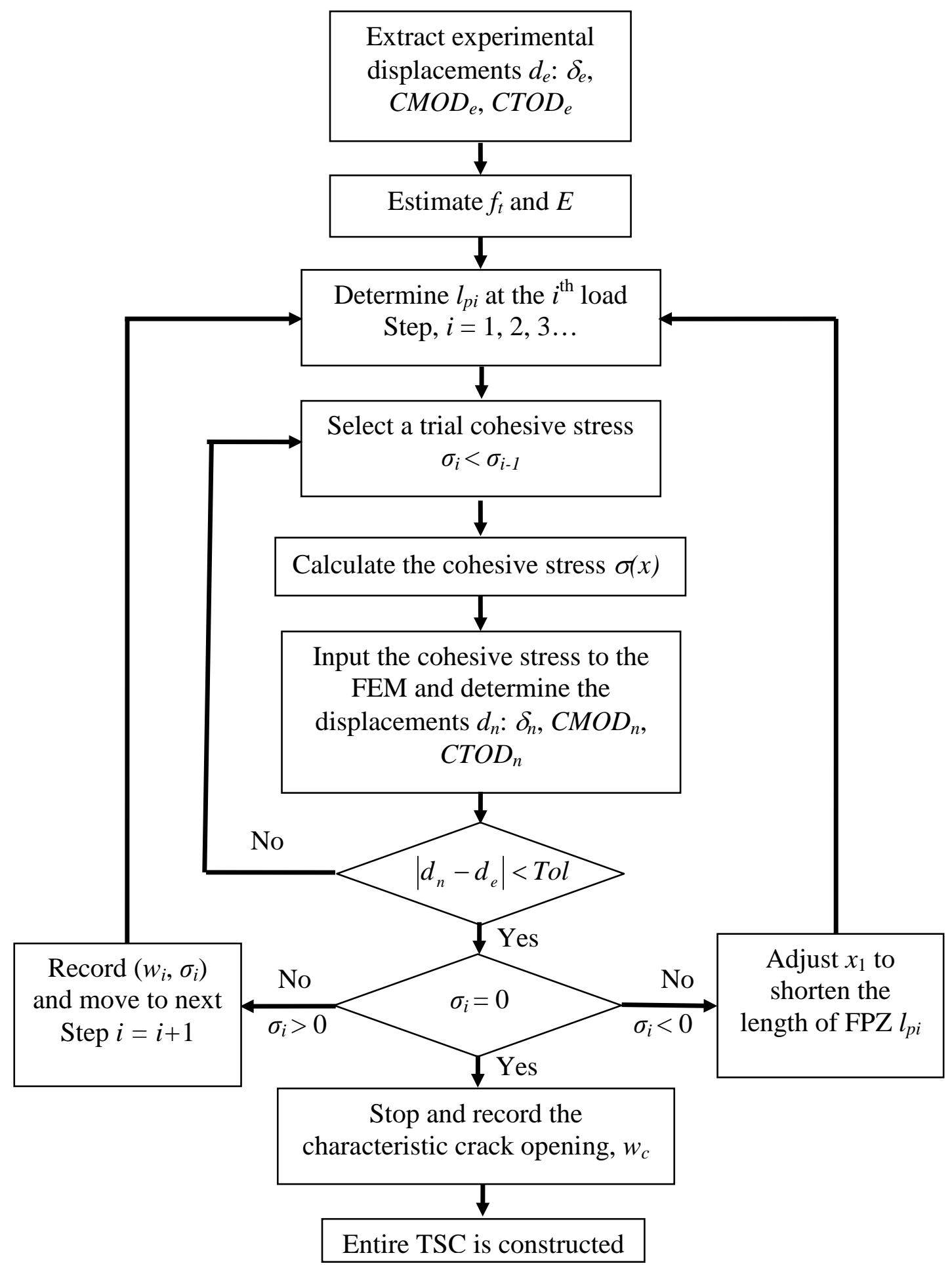

Fig. 3. Flowchart showing the IDCM for the evaluation of the TSC. 

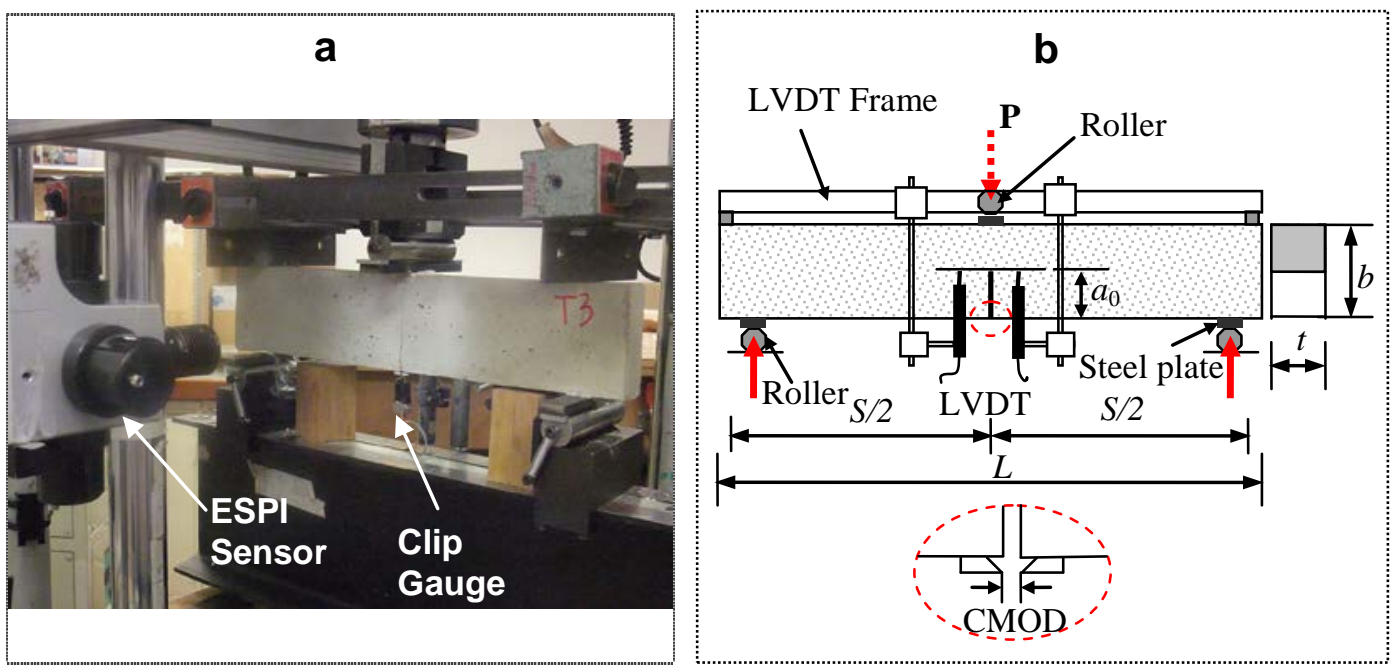

Fig.4. Experimental setup (a) photo and (b) schematic view.
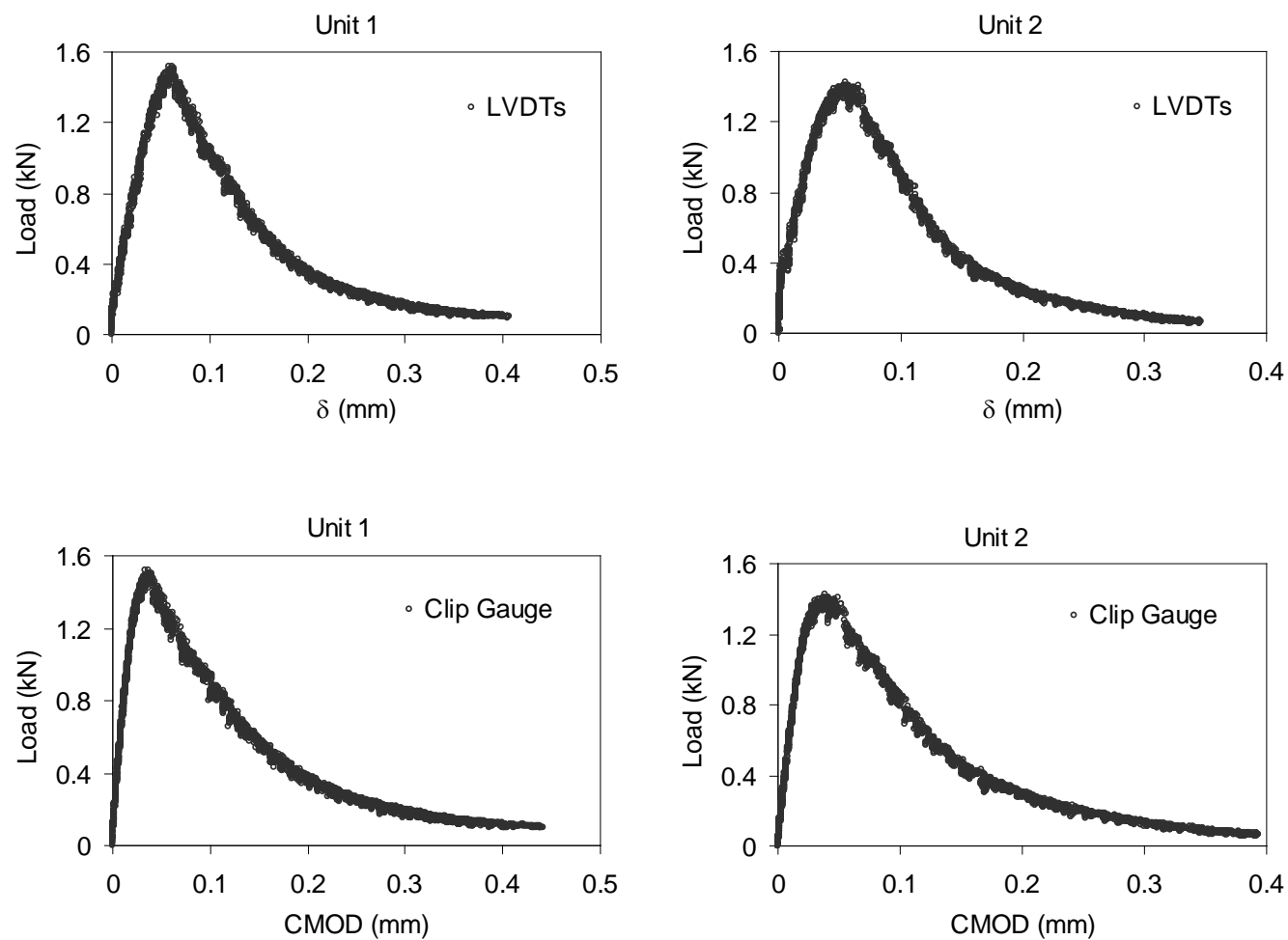

Fig. 5. Experimental $P-\delta$ and $P$-CMOD curves. 
a

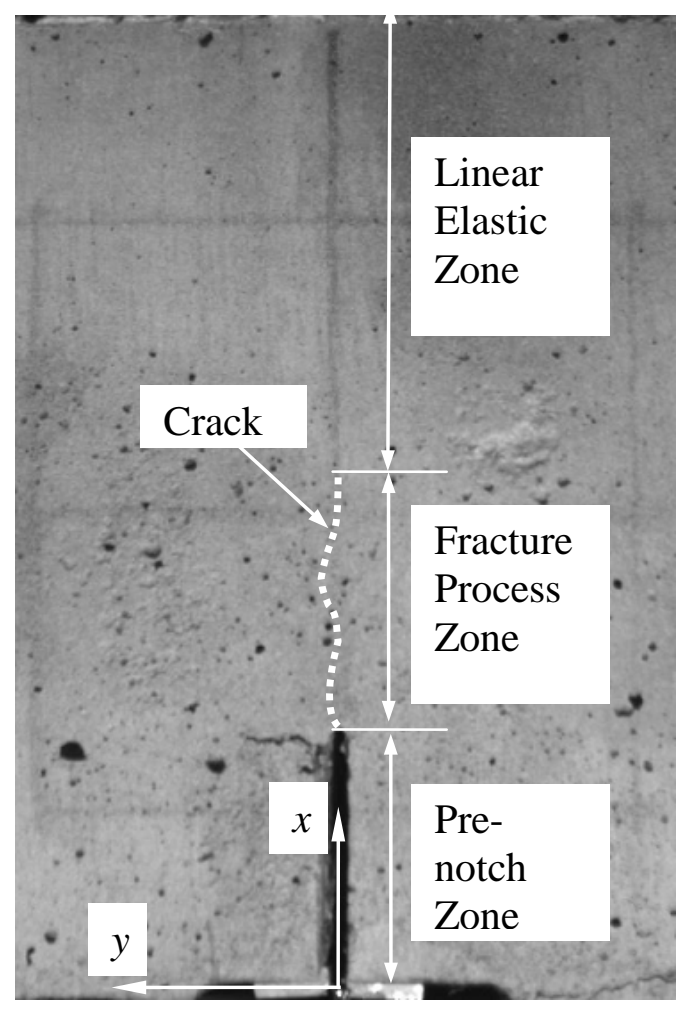

b

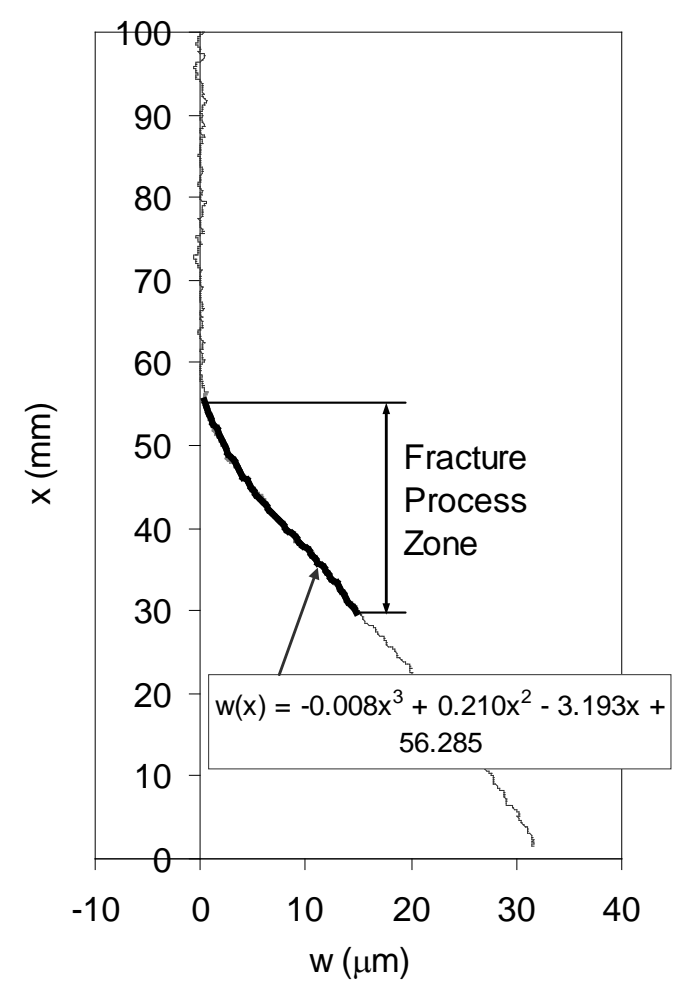

Fig. 6. (a) Measuring surface. (b) Experimental COD profile and polynomial fitting of $w$ in the FPZ. 


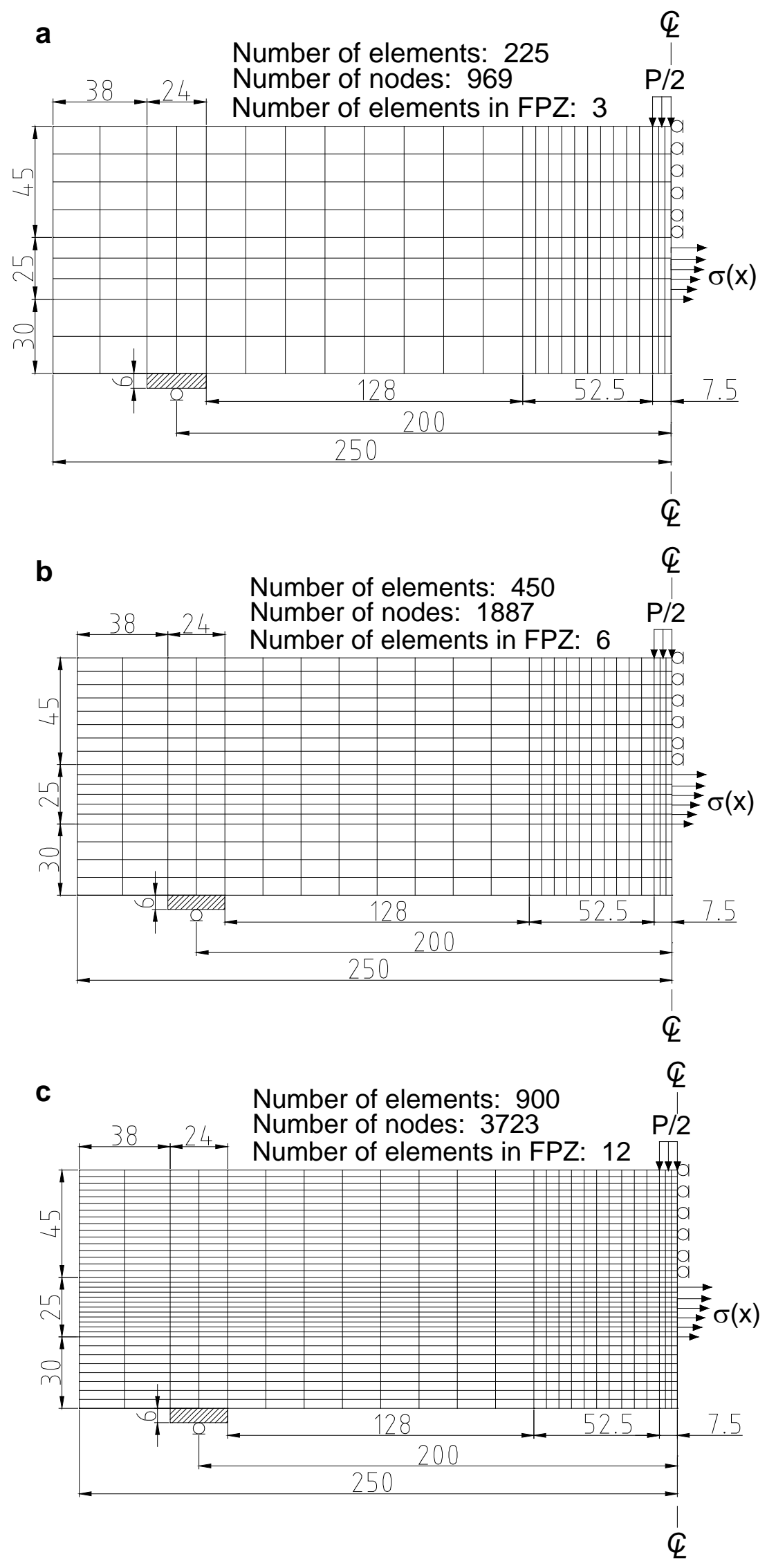

Fig. 7. Specimen configuration and FE meshes: (a) $N=225$; (b) $N=450$; (c) $N=900$. 
a

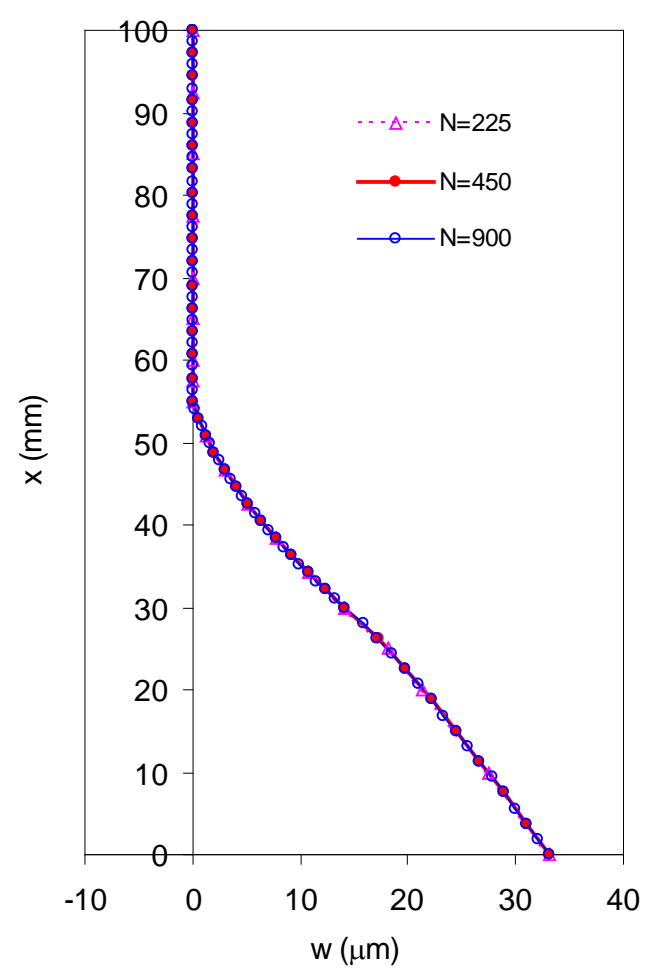

b

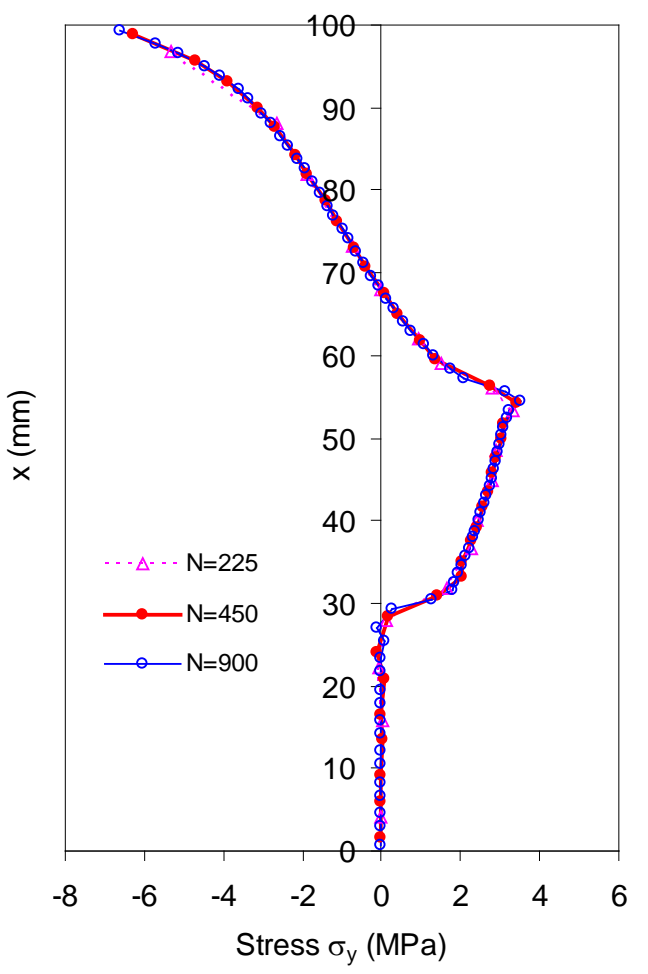

Fig. 8. Effects of mesh density on (a) COD profiles and (b) stress profiles. 
a

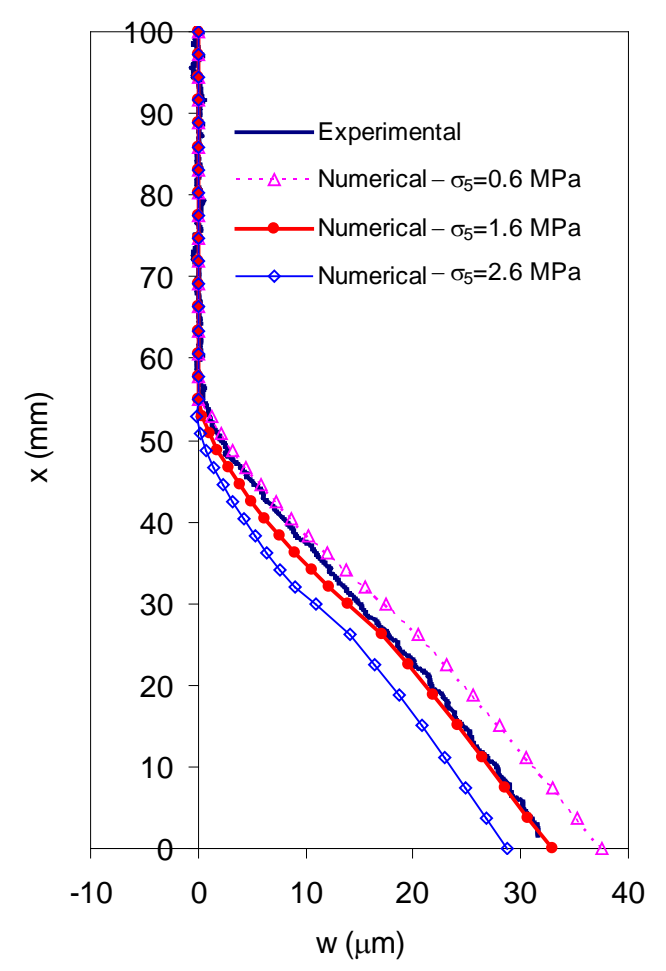

b

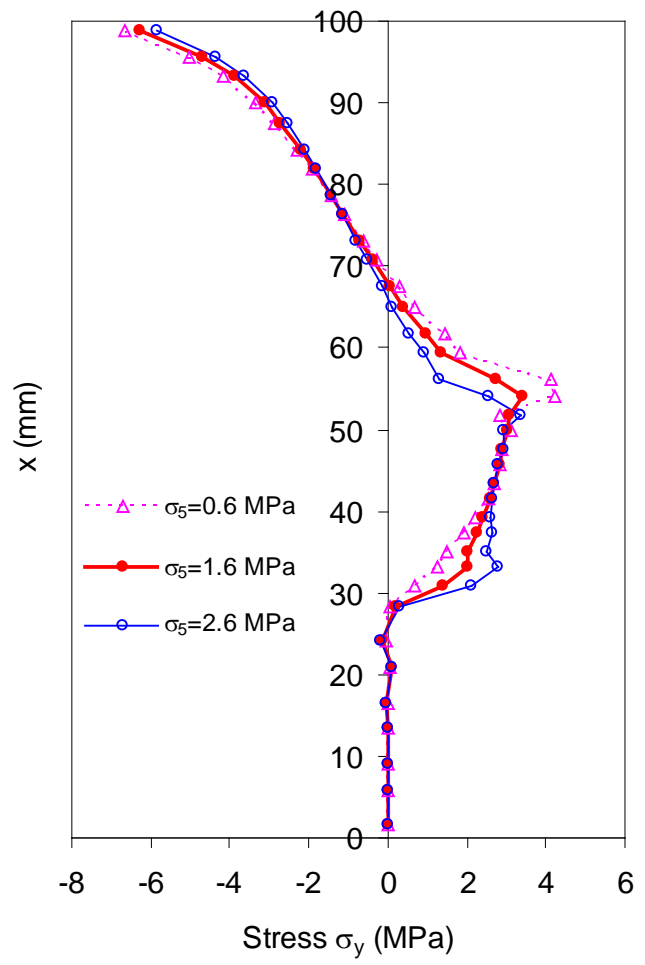

Fig. 9. Effects of the trial cohesive stress $\sigma_{5}$ on (a) COD profiles and (b) stress profiles.

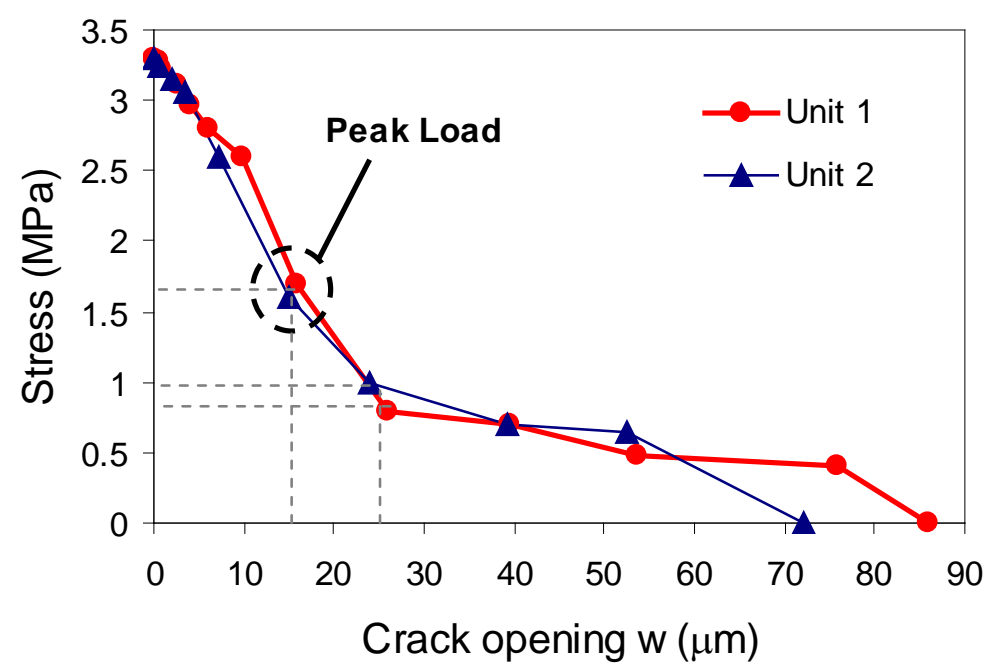

Fig. 10. TSC obtained from the IDCM. 

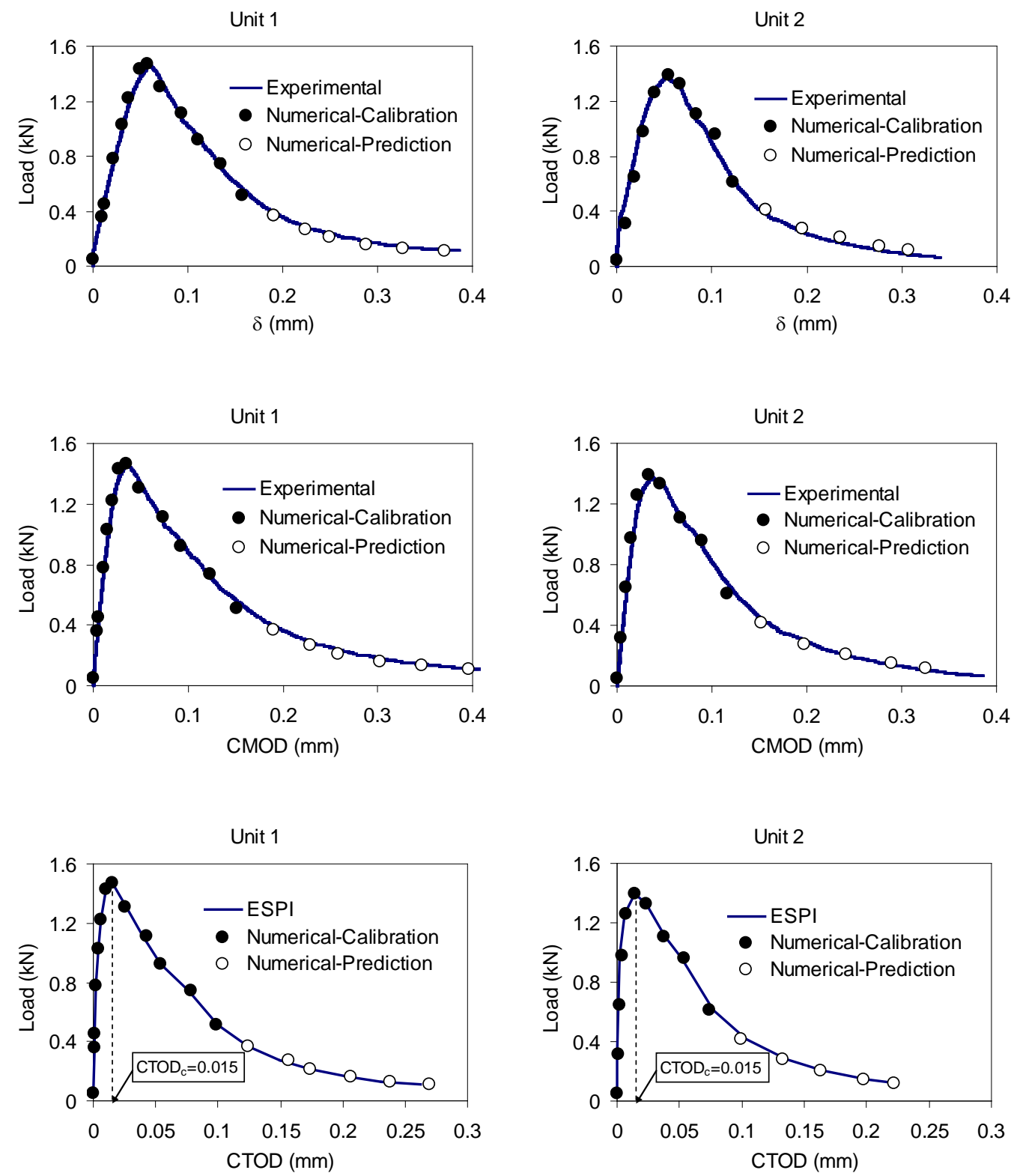

Fig. 11. Comparisons of numerical and experimental $P-\delta$, $P$-CMOD and $P$-CTOD curves. 